\title{
Introducing laparoscopic total gastrectomy for gastric cancer in general practice: a retrospective cohort study based on a nationwide registry database in Japan
}

\author{
Yasuhiro Kodera ${ }^{1} \cdot$ Kazuhiro Yoshida $^{2}$ - Hiraku Kumamaru ${ }^{3}$. Yoshihiro Kakeji ${ }^{4}$. Naoki Hiki ${ }^{5}$. Tsuyoshi Etoh ${ }^{6}$. \\ Michitaka Honda $^{7} \cdot$ Hiroaki Miyata $^{8} \cdot$ Yuichi Yamashita $^{9} \cdot$ Yasuyuki Seto $^{10} \cdot$ Seigo Kitano $^{11} \cdot$ Hiroyuki Konno $^{12}$
}

Received: 17 December 2017 / Accepted: 14 January 2018 / Published online: 9 February 2018

(c) The International Gastric Cancer Association and The Japanese Gastric Cancer Association 2018

\begin{abstract}
Background Although laparoscopic total gastrectomy (LTG) is considered a technically demanding procedure with safety issues, it has been performed in several hospitals in Japan. Data from a nationwide web-based data entry system for surgical procedures (NCD) that started enrollment in 2011 are now available for analysis.

Methods A retrospective cohort study was conducted using data from 32,144 patients who underwent total gastrectomy and were registered in the NCD database between January 2012 and December 2013. Mortality and morbidities were compared between patients who received LTG and those who underwent open total gastrectomy (OTG) in the propensity score-matched Stage I cohort and Stage II-IV cohort.

Results There was no significant difference in mortality rate between LTG and OTG in both cohorts. Operating time was significantly longer in LTG while the blood loss was smaller. In the Stage I cohort, LTG, performed in 33.6\% of the patients, was associated with significantly shorter hospital stay but significantly higher incidence of readmission, reoperation, and anastomotic leakage $(5.4 \%$ vs. $3.6 \%, p<0.01)$. In the Stage II-IV cohort, LTG was performed in only $8.8 \%$ of the patients and was associated with significantly higher incidence of leakage $(5.7 \%$ vs. $3.6 \%, p<0.02)$ although the hospital stay was shorter (15 days vs. 17 days, $p<0.001$ ).

Conclusion LTG was more discreetly introduced than distal gastrectomy, but remained a technically demanding procedure as of 2013. This procedure should be performed only among the well-trained and informed laparoscopic team.
\end{abstract}

Keywords Gastric cancer · Laparoscopic surgery · Open gastrectomy · National Clinical Database · Propensity score matching

Yasuhiro Kodera

ykodera@med.nagoya-y.ac.jp

1 Department of Gastroenterological Surgery, Nagoya University Graduate School of Medicine, Nagoya, Japan

2 Department of Surgical Oncology, Graduate School of Medicine, Gifu University, Gifu, Japan

3 Department of Healthcare Quality Assessment, Graduate School of Medicine, University of Tokyo, Tokyo, Japan

4 Division of Gastrointestinal Surgery, Department of Surgery, Graduate School of Medicine, Kobe University, Kobe, Japan

5 Department of Gastroenterological Surgery, Gastroenterological Center, Cancer Institute Hospital of Japanese Foundation for Cancer Research, Tokyo, Japan
6 Department of Gastroenterological and Pediatric Surgery, Oita University Faculty of Medicine, Oita, Japan

7 Department of Minimally Invasive Medical and Surgical Oncology, Fukushima Medical University, Fukushima, Japan

8 Department of Health Policy and Management, School of Medicine, Keio University, Tokyo, Japan

9 Department of Gastroenterological Surgery, Fukuoka University Faculty of Medicine, Fukuoka, Japan

10 The Japanese Society of Gastroenterological Surgery, Tokyo, Japan

11 Oita University, Oita, Japan

12 Hamamatsu University School of Medicine, Hamamatsu, Japan 


\section{Introduction}

Gastric cancer is the fifth most common malignancy in the world, with 952,000 cases estimated to have occurred in 2012 [1]. Surgery remains the mainstay of treatment with curative intent, and gastrectomy with systemic D2 lymphadenectomy has been arguably the standard procedure in several treatment guidelines [2]. Gastric cancer surgery by the laparoscopic approach has been performed mainly in the East Asia and is reportedly associated with benefits such as faster recovery from the surgical stress, decreased blood loss and shorter hospital stay [3].

Safety of laparoscopic distal gastrectomy for clinically Stage I gastric cancer has already been confirmed in the Japan Clinical Oncology Group (JCOG) 0912 study [4], a prospective phase III study. Subsequently, this approach was acknowledged as an option of standard treatments for this population in the Japanese guidelines [2]. Nevertheless, oncological feasibility of the laparoscopic distal gastrectomy will have to be proven with more advanced gastric cancer in which suboptimal lymph node dissection may compromise survival outcomes. Thus, final survival results of the open versus laparoscopy randomized trials for clinically Stage II/ III gastric cancer conducted in China (the CLASS study [5]), Korea (the KLASS-II study [6]) and Japan (the JLSSG0901 study [7]) are eagerly awaited. As for laparoscopic total gastrectomy (LTG), concerns regarding technically demanding reconstructions that involve the esophagus remain an issue, and a prospective feasibility study to explore safety of this procedure, JCOG1401, is ongoing among high-volume centers in Japan [8]. Despite these concerns, LTG has already begun to be implemented as general practice even in community hospitals.

In the current study, we retrieved and analyzed data of the patients who received total gastrectomy from the National Clinical Database (NCD), a nationwide web-based data entry system for surgical procedures founded in Japan and started the patient enrollment in 2011 [9]. The NCD was selected as the data source so as not to restrict the analysis to data from high-volume or specialized centers as in the case of the JCOG trials, but to reflect the current status of surgical practice in whole of Japan. Comparisons of various relevant short-term outcomes were made between the open and laparoscopic surgery after adjusting for various confounding factors by propensity score matching.

\section{Material and methods}

\section{Data source}

We utilized the data from the Japan Society of Gastroentelogical Surgery (JSGS) registry on the NCD. Since the enrolled cases are linked with a lifelong board certification system for the surgeons, data registration is mandatory not only for the teaching hospitals but also for the community hospitals with surgical departments throughout Japan and, thus, the database expected to cover more than $90 \%$ of the general clinical practice data in general surgery and has become the most comprehensive surgical database in Japan [10]. Audit activities via site visits have been conducted by NCD and JSGS to ensure accuracy of the data, and data obtained from the database have been used for several purposes including establishment of the risk calculators [11] for the benefit of surgical society members as in the case of National Surgery Quality Improvement Program (NSQIP) [12] by the American College of Surgeons. The data components collected for the JSGS registry are almost identical to the NSQIP database.

\section{Patient cohort}

Patients who underwent total gastrectomy for gastric malignancy between January 2012 and December 2013 were retrieved from the NCD registry. Patients were divided into a group with the preoperative diagnosis of Stage I cancer and another group with the diagnosis of Stage II-IV cancer, and these groups were analyzed separately. Data on open total gastrectomy (OTG) for Stage I cancer were recruited from 1489 institutions and laparoscopic total gastrectomy (LTG) from 717 institutions. The median number recruited for each of these procedures was 3 per an institution. Data on OTG for Stage II-IV cancer were recruited from 1777 institutions and LTG from 477 institutions. The median number recruited for the open surgery was 7 per an institution and for the laparoscopic surgery was 2 .

\section{Selection of quality indicators and confounding factors}

We sought to compare the incidence of postoperative complications and other quality indicators between the two surgical approaches. To minimize the effect of several confounding factors, we organized consensus meeting participated by the authors and other supporting personnel to determine the quality indicators of interest and complications that need to be assessed as well as the potential confounding variables. Of the quality indicators, we 
considered 30-day postoperative death and surgical death (deaths that occurred at any time during the hospitalization for surgery) as the most important. Other indicators of interest included occurrences of re-operations within 30 days after surgery, readmissions within 30 days after surgery, operating time, blood loss, and length of postoperative stay. We also assessed the occurrence of complications that were $\geq$ grade 3 based on the classification of Clavien and Dindo [13]. Confounding factors included patients' age, gender, American Society of Anesthesiologists performance status (ASA-PS) score, body mass index (BMI), preoperative conditions including the body weight loss greater than $10 \%$ within the past 6 months, smoking status, whether the surgery was elective or emergent, presence of habitual alcohol intake, the activities of daily living (ADL), and presence of comorbidities such as insulin-dependent diabetes mellitus (DM), respiratory disease, Chronic Obstructive Pulmonary Disease (COPD), hypertension, angina, hemodialysis, congestive heart failure, history of cerebrovascular accident, long-term use of steroids, and bleeding disorders. Factors such as surgical $\mathrm{T}, \mathrm{N}$, and $\mathrm{M}$ categories, presence of concurrent cholecystectomy and splenectomy, and whether the preoperative chemotherapy was given were also considered.

\section{Propensity score matching and statistical analysis}

A biostatistician (H. K.) conducted the propensity score modeling and matching while being blinded to the outcome. The propensity score was estimated using logistic regression models built separately in the cohort of stage I cases and the cohort of Stage II-IV cases, predicting the exposure of undergoing laparoscopic surgery against undergoing open distal gastrectomy from the confounding variables described above. After propensity score estimation, each patient undergoing laparoscopy was matched to a patient undergoing open surgery using a greedy matching algorithm without replacement with a matching caliper of 0.2 standard deviation of logit (propensity score), using the macro presented by Marcelo Coca-Perraillon [14]. The balance of the matched cohort was assessed by calculating the standardized difference between the two groups using the macro by Yang and Dalton [15]. We made comparisons of various outcomes between the matched cohort using Fisher's exact test for the outcomes with expected cell count less than 5, or Pearson's chi-squared test for others for binary variables, and using Wilcoxon rank-sum test for continuous variables. Comparisons were all two-sided and $p$ values less than 0.05 were considered significant. All analyses were conducted using SAS 9.4 (SAS Institute, Cary, NC, USA).

\section{Results}

\section{Patients}

During the two years, 38,868 patients who underwent total gastrectomy had been registered in the NCD. Of these, 1552 patients who did not receive the surgery to treat gastric malignancy, 4222 patients who underwent coresection of organs other than the gall bladder and spleen, and 950 patients whose preoperative clinical stage had not been defined were excluded and the remaining 32,144 patients underwent the analyses (Fig. 1). These patients were subdivided into 11,740 patients with clinically Stage I cancer and 20,404 patients with clinically Stages II-IV cancer, and were analyzed independently.

\section{Patient demographics}

The background characteristics of clinically Stage I patients are shown in Table 1. Of the 11,740 patients, 7793 patients were treated with OTG while 3947 (33.6\%) were operated by the laparoscopic approach. Patients who were treated by OTG tended to be more elderly male patients who tend to have more advanced (Stage IB) disease (32.6\% versus 18.4\%) with greater proportion of the ASA-PS scores of 3-5. A greater proportion of patients treated by OTG underwent cholecystectomy $(24.8 \%$ versus $13.8 \%)$ and splenectomy ( $4.5 \%$ versus $0.1 \%$ ). After the propensity score matching by which 3912 patients who underwent OTG and 3912 who underwent LTG were retrieved, the standardized difference of all these confounding factors was reduced to 0.04 or less (Table 1).

As for more advanced gastric cancer, only 1802 of the 20,404 patients $(8.8 \%)$ were treated by the laparoscopic approach (Table 2). Again, greater proportions of patients treated by OTG underwent cholecystectomy ( $24.1 \%$ versus $12.8 \%$ ) and splenectomy (13\% versus $0.7 \%$ ). In addition, the laparoscopic surgery tended to be selected for cancer of less advanced stage and those without preoperative body weight loss, but the differences in the age, gender, and the incidence of comorbidities between the two groups were not as prominent as in the case of early stage cancers. After the propensity score matching, 1771 patients were retrieved for each approach with improved balance of the confounding factors whose standardized differences were 0.08 or less (Table 2 ). 
Fig. 1 Flow chart of the patients who were registered and analyzed in this study

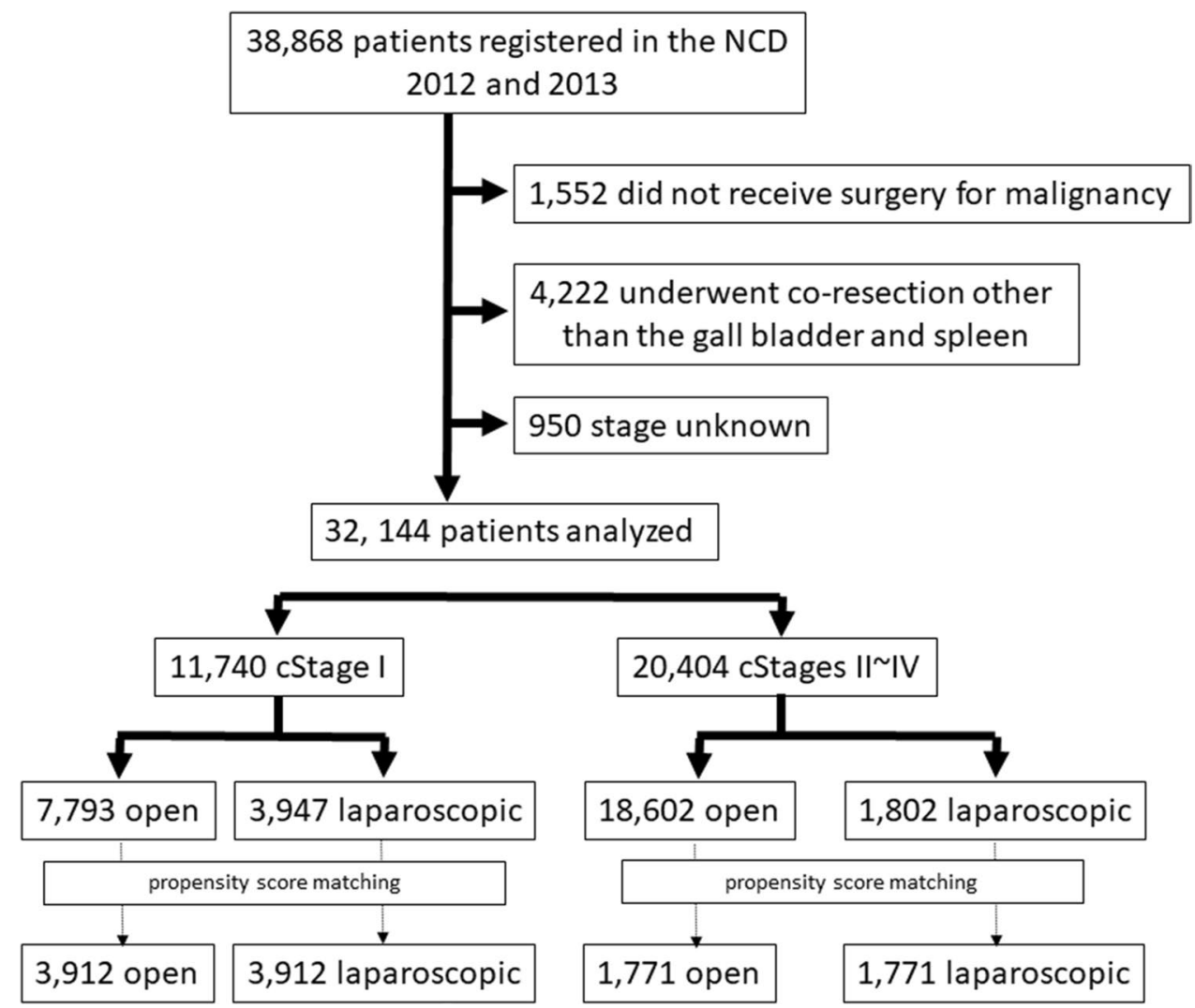

\section{Comparison of morbidity and mortality between the surgical approaches among the Stage I propensity score-matched cohorts (Table 3)}

Operating time was significantly longer in LTG (median time: 349 mins versus 237 mins, $p<0.001)$ whereas the blood loss was significantly smaller (median amount: $76 \mathrm{~mL}$ versus $270 \mathrm{~mL}, p<0.001)$. There was no significant difference in 30-day and in-hospital mortalities, although the in-hospital mortality tended to be slightly higher in the open surgery group $(0.7 \%$ versus $0.5 \%$, $p=0.19)$. The length of hospital stay was significantly longer in the open surgery group at the median of 16 days ( $10^{\text {th }}$ and $90^{\text {th }}$ percentiles $10-34$ day) than the laparoscopic surgery group (median 14 days and $10^{\text {th }}$ and $90^{\text {th }}$ percentiles 9-33 days, $p<0.001$ ), but the incidence of readmission within 30 days after surgery was higher after the laparoscopic surgery $(2.7 \%$ versus $1.7 \%, p=0.002)$. Reoperation within 30 days after surgery was also more frequent among those treated by the laparoscopic surgery (4.5\% versus $3.3 \%, p=0.009)$.

The incidence of postoperative complications tended to be higher after LTG, both regarding all grades $(17.8 \%$ in LTG versus $16.4 \%, p=0.09)$ and $\geq$ grade $3(7.2 \%$ versus $6.2 \%, p=0.08)$, although neither reached statistical significance. Most importantly, there was a significant difference in the incidence of anastomotic leakage (leakage at all anastomotic sites was included) in favor of OTG (3.6\% versus $5.4 \%, p<0.001)$. On the other hand, the incidence of superficial surgical site infection $(2.3 \%$ versus $1.5 \%, p=0.02)$ and pneumonia $(2.7 \%$ versus $2 \%, p=0.03)$ was significantly higher in OTG. There were no differences in the incidence of other complications such as intraabdominal abscess, pancreatic fistula (Grades B and C by the International study group of postoperative pancreatic fistula criteria), wound dehiscence, pulmonary embolism, and sepsis.

\section{Comparison of morbidity and mortality between the surgical approaches in the Stage II-IV propensity score-matched cohorts (Table 4)}

As with the Stage I cohort, the operating time was longer and the amount of blood loss was smaller for the laparoscopic surgery (median time: 349 mins for LTG versus 237 mins, $p<0.001$, median amount: $76 \mathrm{~mL}$ for LTG versus $270 \mathrm{~mL}, p<0.001)$.

There were no significant differences in 30-day and inhospital mortalities. The length of hospital stay was significantly longer in the open surgery group at the median of 17 days $\left(10^{\text {th }}\right.$ and $90^{\text {th }}$ percentiles $10-39$ days) than the LTG group at the median of 15 days $\left(10^{\text {th }}\right.$ and $90^{\text {th }}$ percentiles 9-39 days, $p<0.001)$. There was no difference in the 


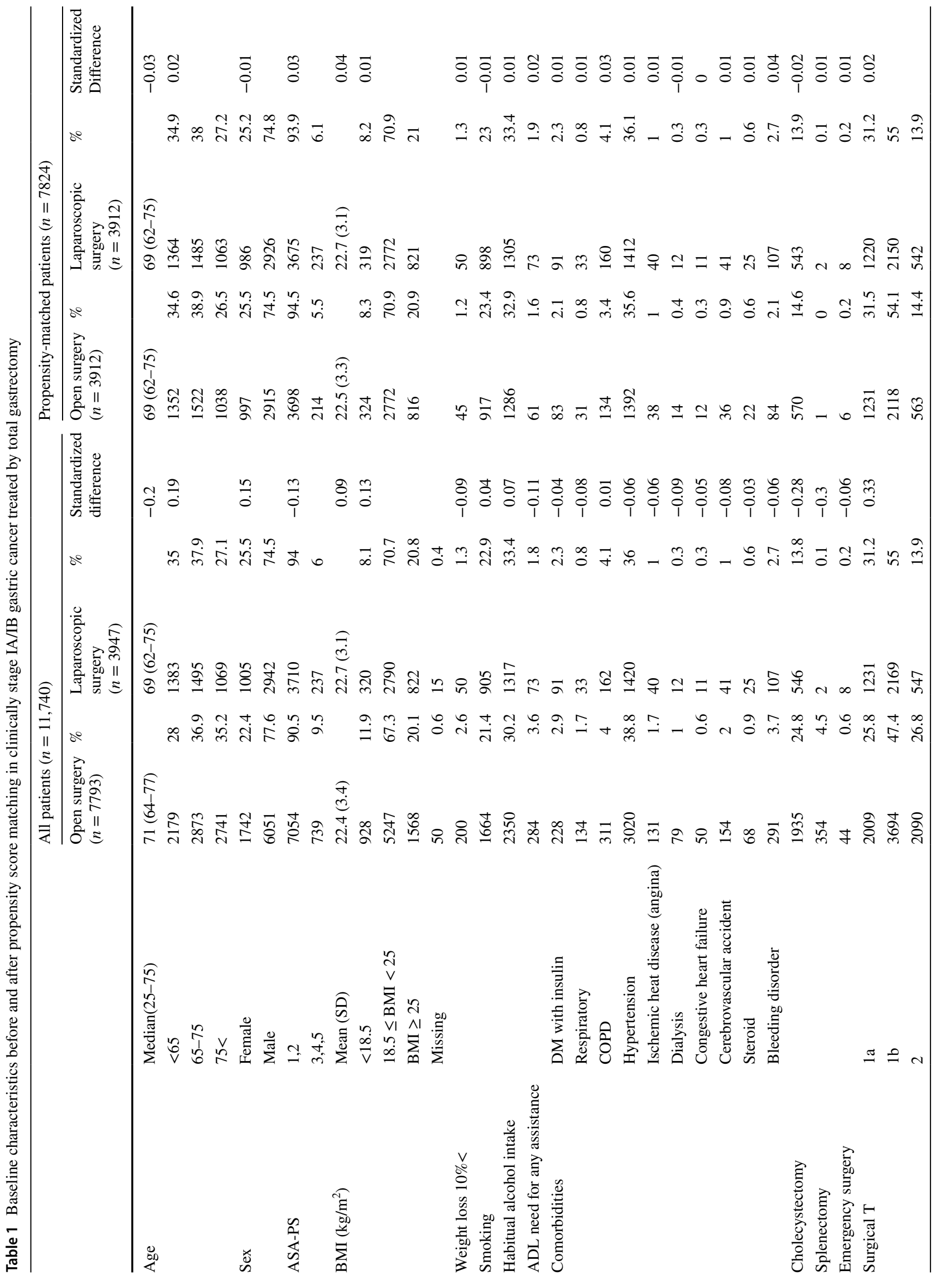




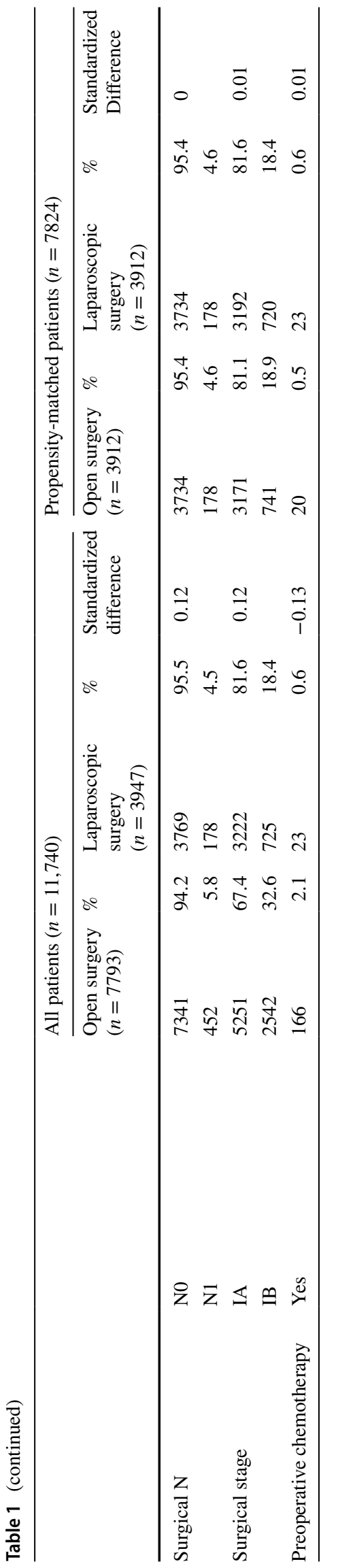

incidence of readmission, but the incidence of reoperation tended to be higher in the laparoscopic surgery $(5.2 \%$ for LTG versus $3.3 \%, p=0.05$ ).

There was no difference in the incidence of any $(20 \%$ in LTG versus $19 \%, p=0.42$ ) or $\geq$ grade 3 (8.6\% in LTG versus $7.4 \%, p=0.17$ ) postoperative complications. However, there was a significant difference in the incidence of anastomotic leakage (leakage at all anastomotic sites were included) in favor of open surgery (3.6\% in open surgery versus $5.7 \%$ in LTG, $p=0.002$ ). On the other hand, the incidence of superficial surgical site infection was significantly higher in open surgery (2.8\% in open surgery versus $1.4 \%$, $p=0.03$ ). Unexpectedly, pneumonia was more frequently observed after the laparoscopic surgery (3.5\% in LTG versus $2 \%, p=0.008)$. There were no differences in the incidence of other complications such as intraabdominal abscess, pancreatic fistula, wound dehiscence, pulmonary embolism, and sepsis.

\section{Discussion}

There had been multitude of articles reporting on singleinstitution experience of LTG performed at specialized centers and by established laparoscopic surgeons [16]. Although most of these report on favorable short-term outcome and conclude that the laparoscopic surgery can be accomplished safely in experienced hands $[16,17]$, some postulate that experience with approximately 100 LTG cases is required to overcome the learning curve [18]. Anastomosis involving the esophagus is associated with technical difficulty [19], whether with the jejunum in case of total gastrectomy or with the remnant stomach in case of proximal gastrectomy. For that reason, several methods of reconstruction, using linear staplers [20,21], circular staplers [22] and orally inserted anvil [23] have been reported, for each of which there are several tips and pitfalls. This is in stark contrast with the situation in open surgery where the use of a circular stapler has been the standard in most circumstances for several decades [24]. LTG has, therefore, been rated by the Japanese guidelines as an investigational procedure that should preferably be conducted on the clinical trial basis, but paradoxically the procedure has been approved by the national insurance system several years ago and the cost for surgery has been reimbursed without strict regulations. Thus, whether the procedure has been adequately introduced to general hospitals and performed safely throughout Japan had been a concern, and the current retrospective analysis of the NCD provided an opportunity to look closely at this issue.

During the two years between January 2012 and December 2013, the laparoscopic approach was selected for 33.6\% of the Stage I gastric cancer patients who were treated by total gastrectomy. This percentage is rather low, considering 


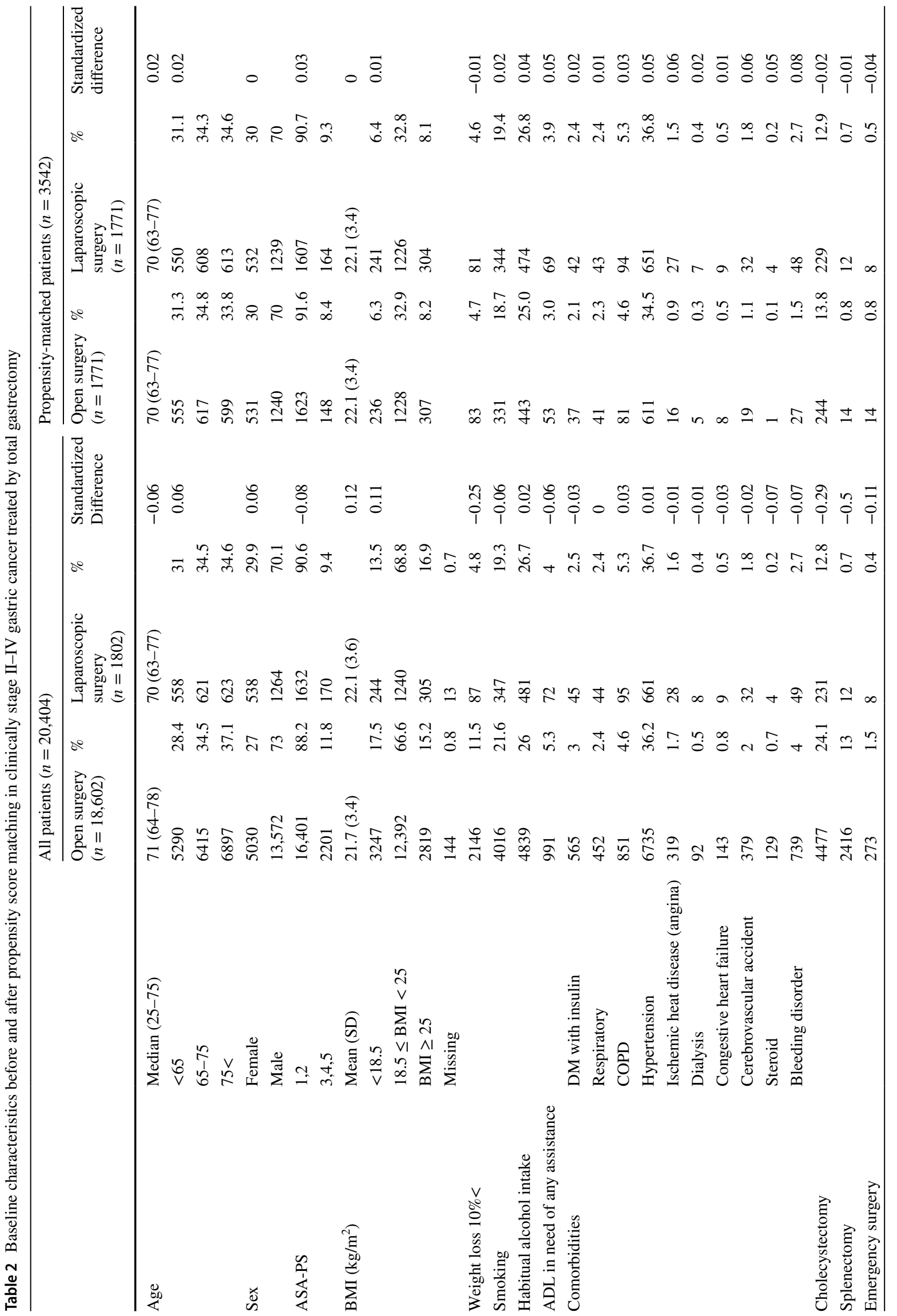




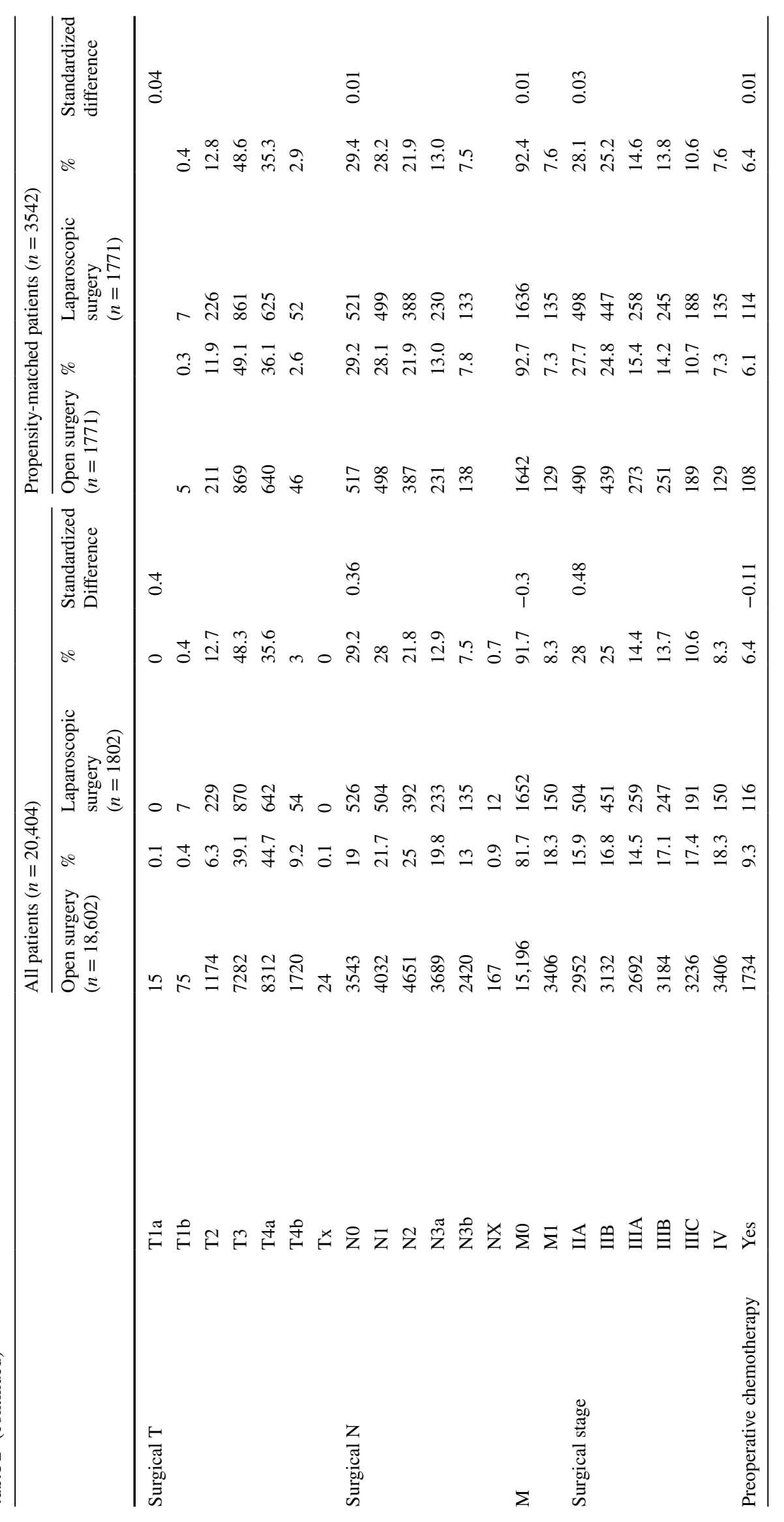


Table 3 Short-term outcome of patients with cStage IA/IB gastric cancer treated by total gastrectomy

\begin{tabular}{|c|c|c|c|c|c|c|}
\hline & & Open surgery $(n=3912)$ & $(\%)$ & $\begin{array}{l}\text { Laparoscopic sur- } \\
\text { gery }(n=3912)\end{array}$ & $(\%)$ & $p$ value \\
\hline Operating time & Median (p10-p90) & $237(153-348)$ & & $349(240-488)$ & & $<0.001$ \\
\hline Blood loss & Median (p10-p90) & $270(71-780)$ & & $76(5-360)$ & & $<0.001$ \\
\hline \multirow[t]{2}{*}{ Mortality } & Within 30 days & 16 & 0.40 & 13 & 0.30 & 0.58 \\
\hline & In-hospital & 28 & 0.70 & 19 & 0.50 & 0.19 \\
\hline Readmission within 30 days & & 66 & 1.70 & 107 & 2.70 & 0.002 \\
\hline Re-operation & & 131 & 3.30 & 176 & 4.50 & 0.009 \\
\hline \multirow[t]{11}{*}{ Complications } & Any complication & 641 & 16.40 & 697 & 17.80 & 0.09 \\
\hline & CD grade 3 and above & 243 & 6.20 & 282 & 7.20 & 0.08 \\
\hline & Superficial SSI & 89 & 2.30 & 60 & 1.50 & 0.02 \\
\hline & Deep SSI & 33 & 0.80 & 33 & 0.80 & 1 \\
\hline & Abscess & 157 & 4 & 185 & 4.70 & 0.12 \\
\hline & Leakage & 141 & 3.60 & 213 & 5.40 & $<0.001$ \\
\hline & Pancreatic fistula (grade B,C) & 57 & 1.50 & 67 & 1.70 & 0.37 \\
\hline & Wound dehiscence & 17 & 0.40 & 9 & 0.20 & 0.12 \\
\hline & Pneumonia & 107 & 2.70 & 77 & 2 & 0.03 \\
\hline & Pulmonary embolism & 3 & 0.10 & 2 & 0.10 & 0.65 \\
\hline & Sepsis & 22 & 0.60 & 24 & 0.60 & 0.76 \\
\hline Length of postoperative & Median (p10-p90) & $16(10-34)$ & & $14(9-33)$ & & $<0.001$ \\
\hline
\end{tabular}

$p$ values derived from Wilcoxon rank-sam test for continuous variables and Pearson's Chi squared or Fisher exact test for binary variables. *median (p10-p90)

Table 4 Short-term outcome of patients with cStage II-IV gastric cancer treated by total gastrectomy

\begin{tabular}{|c|c|c|c|c|c|c|}
\hline & & Open surgery $(n=1771)$ & $(\%)$ & $\begin{array}{l}\text { Laparoscopic sur- } \\
\text { gery }(n=1771)\end{array}$ & $(\%)$ & $p$ value \\
\hline Operating time & Median (p10-p90) & $246(160-360)$ & & $357(236-530)$ & & $<0.001$ \\
\hline Blood loss & Median (p10-p90) & $340(90-946)$ & & $108.5(5-550)$ & & $<0.001$ \\
\hline \multirow[t]{2}{*}{ Mortality } & Within 30 days & 11 & 0.60 & 16 & 0.90 & 0.33 \\
\hline & In-hospital & 32 & 1.80 & 30 & 1.70 & 0.8 \\
\hline Readmission within 30 days & & 36 & 2 & 50 & 2.80 & 0.13 \\
\hline Re-operation & & 58 & 3.30 & 92 & 5.20 & 0.05 \\
\hline \multirow[t]{11}{*}{ Complications } & Any complication & 337 & 19 & 356 & 20.10 & 0.42 \\
\hline & CD grade III and above & 131 & 7.40 & 153 & 8.60 & 0.17 \\
\hline & Superficial SSI & 49 & 2.80 & 24 & 1.40 & 0.03 \\
\hline & Deep SSI & 18 & 1 & 17 & 1 & 0.87 \\
\hline & Abscess & 92 & 5.20 & 104 & 5.90 & 0.38 \\
\hline & Leakage & 63 & 3.60 & 101 & 5.70 & 0.002 \\
\hline & Pancreatic fistula (grade B,C) & 48 & 2.70 & 44 & 2.50 & 0.67 \\
\hline & Wound dehiscence & 12 & 0.70 & 11 & 0.60 & 0.83 \\
\hline & Pneumonia & 36 & 2 & 62 & 3.50 & 0.008 \\
\hline & Pulmonary embolism & 1 & 0.10 & 2 & 0.10 & 0.56 \\
\hline & Sepsis & 11 & 0.60 & 13 & 0.70 & 0.68 \\
\hline Length of postoperative stay & Median (p10-p90) & $17(10-39)$ & & $15(9-39)$ & & $<0.001$ \\
\hline
\end{tabular}

the fact that the laparoscopic approach was selected for as many as $55.4 \%$ of patients with Stage I cancer who underwent distal gastrectomy during the same period [25]. This possibly reflected the technical difficulty as well as the fact that only laparoscopic distal gastrectomy for Stage I cancer is currently recommended as an optional standard procedure 
in the Japanese Gastric Cancer Treatment Guidelines [2]. As for the Stage II-IV cancer, the laparoscopic approach was selected only in $8.8 \%$ of all cases of total gastrectomy. Furthermore, the tendency to prefer the laparoscopic approach for less advanced disease within the cohort was evident both in the Stage I and Stage II-IV cohorts. The laparoscopic approach for Stage I cancer was more commonly selected for younger patients and patients with low ASA-PS scores, but this tendency was not so prominent for the Stage II-IV counterparts. These facts presumably reflect discreet attitude of the Japanese surgeons who have introduced the laparoscopic surgery step-by-step, avoiding the demanding procedures such as esophago-jejunal anastomosis for total gastrectomy or extended lymph node dissection for advanced cancer until they consider they are technically well prepared. In addition, several surgeons seem to have taken the attitude of training themselves primarily by operating laparoscopically on early stage cancers while waiting for emergence of the phase III evidences that are expected to reveal oncological feasibility of the laparoscopic approach in advanced cancers. In contrast, surgeons who performed LTG for advanced cancers were mostly those with extreme expertise in this area and could have already become less selective when choosing patients for the laparoscopic approach during the two years studied.

Given the technical difficulty associated with the esophago-jejunostomy, one of key quality indicators for this study would be the incidence of anastomotic leakage. Indeed, this parameter had been the primary endpoint in the JCOG1401, a one-arm study to confirm the feasibility of laparoscopic total/proximal gastrectomies [8]. Unfortunately, the site of leakage is not specified in the NCD database and may include some cases of leakage at the duodenal stump, although the leakage at the jejunojejunostomy is estimated to be extremely low in incidence. We presumed, nevertheless, that the difference in the incidence of leakage observed in the current study is due to the difference in the most demanding anastomosis, namely at the esophago-jejunostomy. In both the Stage I cohort and the Stage II-IV cohort of the current study, the incidences of anastomotic leakage were significantly higher in the LTG group. However, despite the assumption that only surgeons with supreme expertise in laparoscopic surgery challenged LTG for the Stage II-IV cohort, the incidence of anastomotic leakage was equivalent to that of the Stage I cohort. Moreover, the incidence of reoperation within 30 days from surgery was higher for the laparoscopic approach. This might reflect the possibility that intraabdominal infections including the anastomotic leakage tend to disseminate more extensively after the laparoscopic surgery due to the suppressed formation of intraabdominal adhesions. Nevertheless, the incidence of leakage overall was not as high as it had been feared, considering the fact that the expected and threshold values of the leakage rate in the JCOG1401 trial which was participated only by selected high-volume hospitals had been determined as 3\% and 8\%, respectively [8]. Moreover, the higher incidence of the leakage in the LTG group did not translate into longer hospital stay or higher mortality in either of the cohorts. This could be reflective of the generally shorter postoperative stay among the LTG group in case the postoperative course was uneventful, as well as the fact that the complications had been managed skillfully to avoid untoward consequences in a majority of cases.

More recently, another article by Etoh et al. comparing LTG with OTG using the NCD database was published [26]. In that study, no significant difference was found in the incidence of anastomotic leakage between the two approaches (6.1\% in open surgery versus $5.3 \%$ in laparoscopic surgery, $p=0.59$ ). While virtually entire relevant data recorded in the NCD database during a two-year period from 1804 institutions were retrospectively analyzed in the current study, data were prospectively recorded within 12 months from 169 of 179 institutions that had been invited to participate in that study. The lack of difference in the incidence of leakage in their analysis, then, could in part be due to the selection in the participating institutions, as 10 of the 179 institutions invited to join their study actually refused to participate. Indeed, as many as $37 \%$ of total gastrectomy in their series had been performed by the laparoscopic approach whereas LTG was performed only in $12 \%$ in the current study. On the other hand, that we analyzed data retrieved in 2012-2013 while Etoh et al. analyzed data obtained in 2014 cannot be ignored. A growing number of institutions have began to implement the laparoscopic approach to various types of surgery in recent years, and the time lapse of one year could have resulted in the improvement of outcome.

The current study suffers from inherent limitations due to the retrospective nature. Despite the propensity score matching, the two groups retrieved for comparison between the two approaches in each of the two cohorts are not the same. In addition, the study has weaknesses associated with the limitations in the NCD registration system. Since it was actually not possible to identify the site of anastomotic leakage, a small fraction of events in each group of patients may have involved the duodenal stump rather than the esophagojejunostomy. Neither were the data on complications in the long-term available. Laparoscopic surgery has a good reputation for reducing the incidence of acute bowel obstruction due to the lower frequency of adhesion to the large median incision needed for open surgery, but on the other hand has the inherent problem of internal hernias [27] such as Petersen' herniation [28] that occur due, in turn, to the lack of adhesions. These adverse events could occur at any time after surgery, and follow-up extending to several years is needed to assess their influence on the patients' well-being. 
To conclude, this study has shown quite clearly how the laparoscopic approach was implemented in total gastrectomy for gastric cancer in the early 2010's in Japan, at a time when laparoscopic distal gastrectomy was already widely accepted for treating the early-stage cancer. Surgeons in Japan remained cautious to introduce LTG as a routine practice for clinically Stage I cancer and were even more reluctant to perform LTG for the advanced gastric cancer. Nevertheless, LTG was associated with significantly higher incidence of anastomotic leakage. Surgeons should be aware of the potential risks associated with this surgical procedure and continue to train hard and seek for appropriate assistance from the experts upon introducing this demanding procedure

\section{Compliance with ethical standards}

Conflict of interest Dr. Kodera reports Grants and personal fees from Taiho Pharmaceutical, Grants and personal fees from Chugai Phamaceutical, Grants and personal fees from Sanofi, Grants and personal fees from Merck Serono, Grants and personal fees from Yakult Honsha, Grants and personal fees from Daiichi Sankyo, Grants and personal fees from Otsuka Pharmaceutical Factory, Grants and personal fees from Takeda Pharmaceutical, Grants and personal fees from Johnson \& Johnson, Grants and personal fees from Eli Lilly Japan, Grants from Pfizer Japan, Grants from EA Pharma, Grants and personal fees from ONO Pharmaceutical, Grants and personal fees from Kaken Pharmaceutical, Grants from Covidien Japan, Grants from Shionogi, Grants from Bristol Myers Squib, Grants from Japan Blood Products Organization, Grants from AbbVie GK, Grants from Eizai, Grants from Abbott Japan, Grants from CSL Behring, Grants from Tsumura, Grants from Nippon Kayaku, Grants from Novartis Pharmaceuticals Japan, Grants from KCI, Grants from Toyama Chemical, Grants from Maruho, personal fees from MSD, personal fees from Olympus, personal fees from Asahi Kasei Pharma, outside the submitted work. Dr. Yoshida reports Grants, personal fees and non-financial support from Ono Pharm. Co., Ltd., Grants, personal fees and non-financial support from Taiho Pharm. Co., Ltd., Grants, personal fees and non-financial support from EA Pharma, Grants, personal fees and non-financial support from Sanofi, Grants, personal fees and non-financial support from Yakult Honsha, Grants, personal fees and non-financial support from Chugai Pharm., Grants, personal fees and non-financial support from Takeda Pharm., Grants, personal fees and non-financial support from Lilly Japan, Grants, personal fees and non-financial support from Daiichi Sankyo, Grants, personal fees and non-financial support from Merck Serono, Grants, personal fees and non-financial support from Novartis, Grants from Kyowa Hakko Kirin, outside the submitted work. Hiraku Kumamaru is affiliated to the Department of Healthcare Quality Assessment at the University of Tokyo which is a social collaboration department supported by NCD. The department was formerly supported by endowments from Johnson \& Johnson K.K., Nipro Corporatio, Teijin Pharma Ltd., Kaketsuken K.K., St. Jude Medical Japan Co. Ltd., Novartis Pharma K.K., Taiho Pharmaceutical Co. Ltd., W. L. Gore Associates, Co. Ltd., Olympus Corporation, and Chugai Pharmaceutical Co. Ltd. Hiroaki Miyata is affiliated to the Department of Healthcare Quality Assessment at the University of Tokyo which is a social collaboration department supported by NCD. The department was formerly supported by endowments from Johnson \& Johnson K.K., Nipro Corporation, Teijin Pharma Ltd., Kaketsuken K.K., St. Jude Medical Japan Co. Ltd., Novartis Pharma K.K., Taiho Pharmaceutical Co. Ltd., W. L. Gore Associates, Co. Ltd., Olympus Corporation, and Chugai Pharmaceutical Co. Ltd.. Drs. Kakeji, Hiki, Etoh, Honda, Yamashita, Seto, Kitano and Konno have nothing to disclose.
Ethical approcal This study conforms to the ethical guidelines of the World Medical Association Declaration of Helsinki-Ethical Principles for Medical Research Involving Human Subjects, and written informed consent for surgery and the use of clinical data was obtained from all patients as required by the institutional review boards of all participating institutes.

\section{References}

1. World Health Organization. GLOBOCAN 2012; Estimated Cancer Incidence, Mortality and Prevalence Worldwide in 2012. http:// globocan.iarc.fr/Default.aspx, 2012.

2. Japanese Gastric Cancer Association. Japanese gastric cancer treatment guidelines 2014 (ver. 4). Gastric Cancer. 2017;20(1):1-19.

3. Kodera Y, Fujiwara M, Ohashi N, Nakayama G, Koike M, Morita S, Nakao A. Laparoscopic surgery for gastric cancer: a collective review with meta-analysis of randomized trials. J Am Coll Surg. 2010;211(5):677-86.

4. Katai H, Mizusawa J, Katayama H, Takagi M, Yoshikawa T, Fukagawa T, et al. Short-term surgical outcomes from a phase III study of laparoscopy-assisted versus open distal gastrectomy with nodal dissection for clinical stage IA/IB gastric cancer: Japan Clinical Oncology Group Study JCOG0912. Gastric Cancer 2017;20(4):699-708.

5. Hu Y, Huang C, Sun Y, Su X, Cao H, Hu J, et al. Morbidity and mortality of laparoscopic versus open D2 distal gastrectomy for advanced gastric cancer: a randomized controlled trial. J Clin Oncol. 2016;34(112):1350-7.

6. Hur H, Lee HY, Lee HJ, Kim MC, Hyung WJ, Park YK, et al. Efficacy of laparoscopic subtotal gastrectomy with D2 lymphadenectomy for locally advanced gastric cancer: the protocol of the KLASS-02 multicenter randomized controlled trial. BMC Cancer. 2015; $15: 355$.

7. Inaki N, Etoh T, Ohyama T, Uchiyama K, Katada N, Koeda K, Yoshida K, Takagane A, Kojima K, Sakuramoto S, Shiraishi N, Kitano S. A multi-institutional prospective, phase II feasibility study of laparoscopy-assisted distal gastrectomy with D2 lymph node dissection for locally advanced gastric cancer (JLSSG0901). World J Surg. 2015;39(11):2734-41.

8. Kataoka K, Katai H, Mizusawa J, Katayama H, Nakamura K, Morita S, et al. Non-randomized confirmatory trial of laparoscopy-assisted total gastrectomy and proximal gastrectomy with nodal dissection for clinical Stage I gastric cancer: Japan Clinical Oncology Group Study JCOG1401. J Gastric Cancer. 2016;16(2):93-7.

9. Suzuki H, Gotoh M, Sugihara K, Kitagawa Y, Kimura W, Kondo $\mathrm{S}$, et al. Nationwide survey and establishment of a clinical database for gastrointestinal surgery in Japan: Targeting integration of a cancer registration system and improving the outcome of cancer treatment. Cancer Sci. 2011;102(1):226-30.

10. Gotoh M, Miyata H, Hashimoto H, Wakabayashi G, Konno H, Miyakawa S, et al. National Clinical Database feedback implementation for quality improvement of cancer treatment in Japan: from good to great through transparency. Surg Today. 2016;46(1):38-47.

11. Watanabe M, Miyata H, Gotoh M, Baba H, Kimura W, Tomita N, et al. Total gastrectomy risk model: data from 20,011 Japanese patients in a nationwide internet-based database. Ann Surg. 2014;260(6):1034-9.

12. Cohen ME, Bilimoria KY, Ko CY, Hall BL. Development of an American College of Surgeons National Surgery Quality 
Improvement Program: morbidity and mortality risk calculator for colorectal surgery. J Am Coll Surg. 2009;209(6):1009-16.

13. Clavien PA, Barkun J, de Oliviera ML, Vauthey JN, Dindo D, Schulick RD, et al. The Clavien-Dindo classification of surgical complications: five-year experience. Ann Surg. 2009;250(2):187-96.

14. Coca-Perraillon M. Local and global optimal propensity score matching. SAS Global Forum 2007 Paper 185, 2007.

15. Yang D, Dalton J. A unified approach to measuring the effect size between two groups using SAS. SAS Global Forum 2012 Paper 335, 2012.

16. Kunisaki C, Makino H, Takagawa R, Kimura J, Ota M, Ichikawa $\mathrm{Y}$, et al. A systemic review of laparoscopic total gastrectomy for gastric cancer. Gastric Cancer. 2015;18(2):218-26.

17. Wada N, Kurokawa Y, Takiguchi S, Takahashi T, Yamasaki M, Miyata H, et al. Feasibility of laparoscopy-assisted total gastrectomy in patients with clinical stage I gastric cancer. Gastric Cancer. 2014;17(1):137-40.

18. Jung DH, Son S-Y, Park YS, Shin DJ, Ahn HS, Ahn S-H, et al. The learning curve associated with laparoscopic total gastrectomy. Gastric Cancer. 2016;19(1):264-72.

19. Okabe H, Tsunoda S, Tanaka E, Hisamori S, Kawada H, Sakai Y. Is laparoscopic total gastrectomy a safe operation? A review of various anastomotic techniques and their outcomes. Surg Today. 2015;45(5):549-58.

20. Inaba K, Satoh S, Ishida Y, Taniguchi K, Isogaki J, Kanaya S, Uyama I. Overlap method; novel intracorporeal esophagojejunostomy after laparoscopic total gastrectomy. J Am Coll Surg. 2010;211(6):e25-9.

21. Nagai E, Ohuchida K, Nakata K, Miyasaka Y, Maeyama R, Toma $\mathrm{H}$, et al. Feasibikity and safety of intracorporeal esophagojejunostomy after laparoscopic total gastrectomy: inverted T-shaped anastomosis using linear staplers. Surgery. 2013;153(5):732-8.
22. Matsuda T, Iwasaki T, Mitsutsuji M, Hirata K, Maekawa Y, Tsugawa D, et al. Surgical outcomes of intracorporeal circularstapled esophagojejunostomy using modified over-and-over suture technique in laparoscopic total gastrectomy. Surg Endosc. 2015;29(11):3386-91.

23. Jeong O, Park YK. Intracorporeal circular stapling esophagojejunostomy using the transorally inserted anvil (OrVil) after laparoscopic total gastrectomy. Surg Endosc. 2009;23(11):2624-30.

24. Nomura S, Sasako M, Katai H, Sano T, Maruyama K. Decreasing complication rates with stapled esophagojejunostomy following a learning curve. Gastric Cancer. 2000;3(2):97-101.

25. Yoshida K, Honda M, Kumamaru H, Kodera Y, Kakeji Y, Hiki N, et al. Surgical outcome of laparoscopic distal gastrectomy compared to open distal gastrectomy; a retrospective cohort study based on a nationwide registry database in Japan. Ann Gastroenterol Surg. 2018;2(1):55-64.

26. Etoh T, Honda M, Kumamaru H, Miyata H, Yoshida K, Kodera Y, et al. Morbidity and mortality from a propensity score-matched, prospective cohort study of laparoscopic versus open total gastrectomy for gastric cancer: data from a nationwide web-based database. Surg Endosc. 2017, in press.

27. Yoshikawa S, Shimada M, Kurita N, Sato H, Iwata T, Higashijima J, et al. Characteristics of internal hernia after gastrectomy with Roux-en-Y reconstruction for gastric cancer. Surg Endosc. 2014;40(3):330-7.

28. Kojima K, Inokuchi M, Kato K, Motoyama K, Sugihara K. Petersen's hernia after laparoscopic distal gastrectomy with Roux-en-Y reconstruction for gastric cancer. Gastric Cancer. 2014;17(1):146-51. 